\title{
Optimal Contest Design when the Designer's Payoff Depends on Competitive Balance
}

\author{
Marco Runkel ${ }^{*}$ \\ University of Munich \\ Department of Economics \\ Ludwigstr. 28, Vgb., III \\ D-80539 Munich \\ Germany \\ phone: ++49(0) 8921806339 \\ fax: ++49 (0) 8921803128 \\ e-mail: marco.runkel@lrz.uni-muenchen.de
}

30th January 2004

\begin{abstract}
This paper investigates optimal contest design when the designer's payoff is increasing in competitive balance between contestants. A two-player contest with asymmetric effort costs (asymmetric abilities) is considered. Competitive balance is measured by the difference in winning probabilities of the contestants. In contrast to previous studies, the impact of competitive balance on the optimal prize is not unique, but depends on the shape of the contest success function. Furthermore, it is shown that including competitive balance in the designer's objective may induce the designer to increase the contestants' effort costs, for example, by tightening the rules of the contest.
\end{abstract}

JEL classification: $\quad$ D72, C72

key words: $\quad$ Optimal contest design, Competitive balance

*I would like to thank Ulrich Woitek for helpful comments. All errors remain my own. 


\section{Introduction}

Lobbying, sports events or promotions can be viewed as contests in which competitors spend resources in hope of winning a prize. There is a large body of literature on the optimal design of contests. Previous authors investigate, for example, contest design as a principal-agent problem (Singh and Wittman, 2001), the optimal choice of several prizes (Glazer and Hassin, 1988) and the optimal design of rent-seeking contests (Kohli and Singh, 1999). Other studies look at the optimal contest success function (Dasgupta and Nti, 1998) and the optimal structure of multi-stage contests (Gradstein, 1998, Gradstein and Konrad, 1999, Amegashie, 1999, 2000). The contest designer is usually supposed to maximize expected profit which equals expected total output (performance) of the contestants less payments (prizes) to the contestants. ${ }^{1}$

An important aspect in many contest design problems is that a close contest is often more exciting for the audience and attracts more attention than a unbalanced contest. The designer's payoff is then increasing not only in the contestants' performance, but also in the closeness of the contest or, in other words, in competitive balance between contestants. Obvious examples are sports or song contests which are financed by advertising or admission fees. The closer such a contest is, the larger is the viewers' and the firms' willingness-to-pay for watching and sponsoring it and the larger is the designer's revenue. Less obvious but also important examples can be found in the political economy of fiscal federalism, e.g. if the central government allocates grants to regional governments which lobby for the grants. The voters represent the 'audience' in this contest and the central government should care about 'balanced' lobbying, since otherwise it may be punished by voters in the next election.

An interesting study of the implications of competitive balance for optimal contest design is provided by Singh and Wittman (1998). They consider a two-player contest with private information about the abilities of the players. The designer chooses the

\footnotetext{
${ }^{1}$ Gradstein (1998) and Gradstein and Konrad (1999) focus on effort-maximizing contests, since in their models a contestant's output is determined by effort and the prize is fixed. Dasgupta and Nti (1998) additionally take into account that the designer herself may value the prize. In rent-seeking contests, the designer is often assumed to minimize effort expenditures of the contestants, since these expenditures are regarded social waste (e.g. Amegashie, 1999, 2000).
} 
prize awarded to the winner. Her objective is increasing in total performance of the contestants and in competitive balance. The latter is measured by the difference in the contestants' performance. The optimal prize is shown to fall short of the optimal prize in case the designer's payoff depends on total performance only. ${ }^{2}$

The paper at hand further investigates optimal contest design when the designer's objective is influenced by competitive balance. A contest model is presented which differs from the Singh-Wittman model mainly in two respects. First, competitive balance is not measured by the difference in performances. In the view of spectators, a contest is close if the outcome is uncertain and this uncertainty is the highest if all players have an equal chance of winning. We therefore measure competitive balance by the difference in winning probabilities of the contestants. It will be shown that, in general, both measures are not equivalent. Second, changing the measure of competitive balance complicates the analysis. In order to keep the analysis tractable, the assumption of asymmetric information is therefore dropped. Contestants are assumed to differ in abilities or, equivalently, in effort costs which are common knowledge. A symmetric information structure is assumed by many of the authors mentioned above.

Under these alternative assumptions, the first aim of the paper is to reexamine how the inclusion of competitive balance in the designer's objective changes the optimal prize. The result turns out to depend on the shape of the so-called contest success function (CSF) which links a contestant's winning probability to effort levels of all contestants. For a specific functional form of the CSF, including competitive balance in the designer's objective lowers the optimal prize. This is in line with the result of the Singh-Wittman model. But other specifications of the CSF lead to the opposite result. Moreover, we fully characterize the class of CSFs for which the optimal prize is independent of competitive balance. This independence result is of special interest, since the class of CSFs derived contains the power function which is frequently used in contest theory (e.g. Tullock, 1980, Gradstein and Konrad, 1999, Amegashie, 2000).

Besides the optimal prize policy, the second aim of the paper is to investigate the designer's optimal regulation of the contestants' effort costs. It is assumed that the

\footnotetext{
${ }^{2} \mathrm{~A}$ discrete version of this model is analyzed in Singh and Wittman (1988). Equity aspects are also discussed in the interest group model of Kohli and Singh (2001). But their framework is quite different from the model of Singh and Wittman (1998) and the one presented here.
} 
designer can increase effort costs, but that it is impossible to discriminate between contestants. Cost regulation of this kind can be interpreted as a tightening of the contest rules. It is observed, for example, in contests where the contestants use technical equipment like cars in motor racing. Another interpretation of non-discriminatory cost regulation is the prohibition of doping. Intensifying doping controls increases the expected cost of doping for all contestants. Our analysis shows that there is no scope for cost regulation, if the designer's objective depends on performance only. Tightening the contest rules harms performance of the contestants and decreases the designer's payoff. However, reducing the contestants' abilities may render the contest closer. Cost regulation may therefore be optimal, if competitive balance matters.

It should be noted that competitive balance of contests is also discussed in the rapidly growing literature on sport economics. For excellent surveys see Fort and Quirk (1995) and Szymanski (2003). But the focus in this literature slightly differs from the one considered here. A great part of the articles is not concerned with general prize and cost regulation, but investigates specific sport policy instruments like e.g. revenue sharing or rookie draft. Those studies which focus on prize and cost policy usually investigate other kinds of this policy as, for example, the division of a given trophy money on a first and second prize as in Szymanski and Valletti (2002). Moreover, a systematic analysis of the impact of different CSFs on optimal contest design in the presence of competitive balance is missing also in the sport economic literature.

The paper is organized as follows. Section 2 presents the contest model, derives an equilibrium and conducts comparative statics. Sections 3 and 4 consider the designer's optimal prize policy and cost regulation, respectively. In Section 5, the designer determines both the prize and the cost regulation parameter. Section 6 concludes.

\section{Asymmetric Contest}

Consider a contest with two risk-neutral contestants competing for a prize $v>0$. The contestants take the prize as given. Contestant $i=1,2$ expends effort $x_{i} \geq 0$ in hope of winning the prize. The probability that contestant $i$ wins the contest is

$$
P^{i}\left(x_{i}, x_{j}\right)=\frac{H\left(x_{i}\right)}{H\left(x_{i}\right)+H\left(x_{j}\right)}, \quad i \neq j
$$


with $H(x) \geq 0$ and $H^{\prime}(x)>0$ for all $x \geq 0 . H(x)$ can be interpreted as the output or performance of a contestant who expends effort $x$. The winning probability of contestant $i$ then equals the ratio of $i$ 's performance to total performance of both players. Skaperdas (1996) refers to $P^{i}$ as the contest success function (CSF), while in Dasgupta and Nti (1998) the function $H$ represents the CSF. We refer to both $P^{i}$ and $H$ as CSF, since $P^{i}$ is determined by $H$ alone. The specification in (1) ensures that the CSF satisfies the axioms (A1) to (A5) imposed by Skaperdas (1996).

Contestants differ in the costs of effort. Unit cost of contestant $i$ is $c_{i}>0$. Contestant 1 is the low-cost player and contestant 2 the high-cost player, i.e. $c_{1}<c_{2}$. In addition, the contestants' unit costs are influenced by a uniform cost parameter $\tau$. This parameter is under the control of the contest designer and is taken as given by the contestants. Total effort cost of contestant $i$ is $\left(c_{i}+\tau\right) x_{i}$. Contestant $i$ 's expected profit equals the expected prize less effort cost, i.e.

$$
\Pi^{i}\left(x_{i}, x_{j}\right)=P^{i}\left(x_{i}, x_{j}\right) v-\left(c_{i}+\tau\right) x_{i}, \quad i \neq j
$$

Notice that such a contest with asymmetric costs is equivalent to a contest with asymmetric abilities. To see this, consider a contest with the CSF $H\left[a_{i}(\tau) e_{i}\right]$ where $e_{i}$ represents contestant $i$ 's effort expenditure and $a_{i}(\tau)$ indicates $i$ 's ability as a function of the parameter $\tau$. Profit of contestant $i$ is then $\widetilde{\Pi}^{i}\left(e_{i}, e_{j}\right)=P^{i}\left[a_{i}(\tau) e_{i}, a_{j}(\tau) e_{j}\right] v-e_{i}$. This profit function is equivalent to (2), if we define $x_{i}:=a_{i}(\tau) e_{i}$ and $a_{i}(\tau):=1 /\left(c_{i}+\tau\right)$. Hence, the contest with asymmetric costs can be interpreted as a contest with asymmetric abilities, where contestant 1 is more able than contestant 2 and an increase in $\tau$ harms the contestants' abilities to transform effort into performance.

The first- and second-order conditions for an interior (pure-strategy) Nash equilibrium of the contest are, respectively,

$$
\begin{gathered}
\Pi_{x_{i}}^{i}\left(x_{i}, x_{j}\right)=\frac{H^{\prime}\left(x_{i}\right) H\left(x_{j}\right)}{\left[H\left(x_{i}\right)+H\left(x_{j}\right)\right]^{2}} v-\left(c_{i}+\tau\right)=0, \quad i \neq j, \\
\Pi_{x_{i} x_{i}}^{i}\left(x_{i}, x_{j}\right)=\frac{H^{\prime \prime}\left(x_{i}\right)\left[H\left(x_{i}\right)+H\left(x_{j}\right)\right]-2\left[H^{\prime}\left(x_{i}\right)\right]^{2}}{\left[H\left(x_{i}\right)+H\left(x_{j}\right)\right]^{3}} H\left(x_{j}\right) v<0, \quad i \neq j .
\end{gathered}
$$

It is assumed throughout that an equilibrium exists so that (3) and (4) are satisfied and the equilibrium profits of both contestants are positive. Equilibrium effort levels are functions of $v$ and $\tau$, i.e. $x_{1}^{*}=X^{1}(v, \tau)$ and $x_{2}^{*}=X^{2}(v, \tau)$. Whether an equilibrium 
really exists, depends on the shape of the CSF. Existence will therefore explicitly be proven when we introduce specific functional forms of the CSF (see footnote 4 and 5). For the time being, we only need the condition

$$
H(x) H^{\prime \prime}(x)-\left[H^{\prime}(x)\right]^{2}<0 \quad \text { for all } \quad x \geq 0
$$

which is necessary for the existence of an equilibrium. ${ }^{3}$ This can be proven by contradiction. Suppose an equilibrium exists and (5) is not satisfied. Equation (3) yields $\left(c_{2}+\tau\right) H^{\prime}\left(x_{1}^{*}\right) H\left(x_{2}^{*}\right)=\left(c_{1}+\tau\right) H^{\prime}\left(x_{2}^{*}\right) H\left(x_{1}^{*}\right) . \quad c_{1}<c_{2}$ implies $H^{\prime}\left(x_{1}^{*}\right) / H\left(x_{1}^{*}\right)<$ $H^{\prime}\left(x_{2}^{*}\right) / H\left(x_{2}^{*}\right)$. If (5) is not satisfied, $H^{\prime}(x) / H(x)$ is non-decreasing in $x$ and we obtain $x_{1}^{*} \leq x_{2}^{*}$. But it can then be shown that $\Pi_{x_{1} x_{1}}^{1}\left(x_{1}^{*}, x_{2}^{*}\right)>0$, i.e. contestant 1 does not attain a profit maximum and there does not exist an equilibrium. This contradicts the initial assumption. Hence, if an equilibrium exists, condition (5) has to be satisfied. $H^{\prime}(x) / H(x)$ is then decreasing in $x$ and we obtain $x_{1}^{*}>x_{2}^{*}$. This immediately implies

Proposition 1. Equilibrium effort, performance and winning probability are larger for the low-cost contestant than for the high-cost contestant for all $v$ and $\tau$.

The intuition of Proposition 1 is obvious. Contestant 1 is more able than contestant 2, since she achieves every given effort level at lower cost. She employs this cost advantage in order to realize higher effort and performance with the consequence that her chance of winning is larger than for contestant 2. Using the terminology of Baik (1994) and Nti (1999b), contestant 1 is the favorite and contestant 2 the underdog.

For further use, a comparative static analysis of the Nash equilibrium is needed. Of interest is the impact of $v$ and $\tau$ on equilibrium effort levels $x_{i}^{*}=X^{i}(v, \tau)$, aggregated performance and competitive balance. Aggregated performance is defined as $a=H\left(x_{1}\right)+H\left(x_{2}\right)$. In equilibrium, it becomes

$$
a^{*}=H\left[X^{1}(v, \tau)\right]+H\left[X^{2}(v, \tau)\right]=: A(v, \tau) .
$$

A natural measure of competitive balance is the variance of the contestants' winning probabilities. The more equally distributed winning probabilities are, the closer is the contest and the better is competitive balance. In a two-player contest, the mean of

\footnotetext{
${ }^{3}$ It is also necessary for the stability condition derived by Nti (1999a, Proposition 3).
} 
winning probabilities is $1 / 2$ and the variance reads $\sigma^{2}=\sum_{i=1}^{2}\left(P^{i}-1 / 2\right)^{2} / 2$. Taking into account $P^{1}+P^{2}=1$, it is straightforward to show that the variance equals $\sigma^{2}=\left(P^{1}-P^{2}\right)^{2} / 4$. The standard deviation of winning probabilities is $\sigma=\left(P^{1}-P^{2}\right) / 2$. Competitive balance may thus be measured by the difference in winning probabilities, $b=P^{1}\left(x_{1}, x_{2}\right)-P^{2}\left(x_{2}, x_{1}\right)$. In equilibrium, this difference can be written as

$$
b^{*}=\frac{H\left[X^{1}(v, \tau)\right]-H\left[X^{2}(v, \tau)\right]}{H\left[X^{1}(v, \tau)\right]+H\left[X^{2}(v, \tau)\right]}=: B(v, \tau)
$$

Proposition 1 implies $b^{*}>0$ for all $v$ and $\tau$. If $b^{*}$ increases (decreases), then equilibrium competitive balance is worsened (improved). Notice that measuring competitive balance by the difference in performances, $q^{*}=H\left(x_{1}^{*}\right)-H\left(x_{2}^{*}\right)$, is not equivalent to measuring competitive balance by $b^{*}$. If the favorite expends more (less) effort and the underdog less (more), then both $q^{*}$ and $b^{*}$ increase (decrease). But if effort levels move into the same direction, then it is possible that $b^{*}$ rises while $q^{*}$ falls et vice versa.

Totally differentiating the first-order conditions (3) yields the Jacobian determinant

$$
\Delta=\frac{v^{2} H_{1} H_{2}}{\left(H_{1}+H_{2}\right)^{6}} \prod_{i \neq j}\left[H_{i}^{\prime \prime}\left(H_{i}+H_{j}\right)-2\left(H_{i}^{\prime}\right)^{2}\right]+\frac{v^{2}\left(H_{1}^{\prime} H_{2}^{\prime}\right)^{2}\left(H_{1}-H_{2}\right)^{2}}{\left(H_{1}+H_{2}\right)^{6}}>0,
$$

where, for notational convenience, $H_{i}:=H\left(x_{i}^{*}\right), H_{i}^{\prime}:=H^{\prime}\left(x_{i}^{*}\right)$ and so on. The sign of the Jacobian follows from the second-order conditions (4). With respect to the prize $v$, the following comparative static results are obtained:

$$
\begin{aligned}
& X_{v}^{i}(v, \tau)=\frac{v H_{i} H_{i}^{\prime}\left[\left(H_{j}^{\prime}\right)^{2}-H_{j} H_{j}^{\prime \prime}\right]}{\Delta\left(H_{i}+H_{j}\right)^{4}}, \quad i \neq j, \\
& A_{v}(v, \tau)=H_{1}^{\prime} X_{v}^{1}(v, \tau)+H_{2}^{\prime} X_{v}^{2}(v, \tau), \\
& B_{v}(v, \tau)=\frac{2 v H_{1} H_{2}\left(H_{1}^{\prime} H_{2}^{\prime}\right)^{2}}{\Delta\left(H_{1}+H_{2}\right)^{6}}\left[F\left(x_{1}^{*}\right)-F\left(x_{2}^{*}\right)\right],
\end{aligned}
$$

where the subscript $v$ denotes the partial derivative with respect to the prize and

$$
F(x):=\frac{H(x) H^{\prime \prime}(x)}{\left[H^{\prime}(x)\right]^{2}}
$$

Attention is restricted to CSFs for which $F(x)$ is monotone. (7) to (9) then yield ${ }^{4}$

\footnotetext{
${ }^{4}$ The CSF (10) is twice differentiable and satisfies $H(0)=0, H^{\prime}(x)>0$ and $H^{\prime \prime}(x)<0$ for all
} 
Proposition 2. (a) An increase in the prize $v$ enhances equilibrium effort and performance of both contestants and equilibrium aggregated performance.

(b) An increase in the prize $v$ improves equilibrium competitive balance if

$$
H(x)=k \ln (1+x) \quad \text { with } \quad k>0 .
$$

It worsens equilibrium competitive balance if

$$
\left.H(x)=\exp \left\{x^{k}\right\} \quad \text { with } \quad k \in\right] 0,1[\text {. }
$$

Equilibrium competitive balance is independent of the prize $v$ if and only if

$$
H(x)=\left[\left(1-k_{1}\right) k_{2} x+\left(1-k_{1}\right) k_{3}\right]^{\frac{1}{1-k_{1}}} \quad \text { with } \quad k_{1}<1, k_{2}>0, k_{3} \geq 0 .
$$

Proof: Taking into account (5) in (7) yields $X_{v}^{i}(v, \tau)>0$ which by (8) implies $A_{v}(v, \tau)>0$. This together with $H^{\prime}>0$ proves (a). For proving (b), suppose first $H$ takes on the functional form (10). Then $F(x)=-\ln (1+x)$ and $F^{\prime}(x)=-1 /(1+x)<0$. $x_{1}^{*}>x_{2}^{*}$ implies $F\left(x_{1}^{*}\right)<F\left(x_{2}^{*}\right)$ and $B_{v}(v, \tau)<0$, i.e. an increase in $v$ improves competitive balance. If $H$ is defined by $(11)$, then $F(x)=\left(k-1+k x^{k}\right) / k x^{k}$ and $F^{\prime}(x)=-(k-1) / x^{k+1}>0$ according to $\left.k \in\right] 0,1\left[\right.$. Taking into account $x_{1}^{*}>x_{2}^{*}$ yields $F\left(x_{1}^{*}\right)>F\left(x_{2}^{*}\right)$ and $B_{v}(v, \tau)>0$, i.e. an increase in $v$ reduces competitive balance.

It remains to prove under which conditions competitive balance is independent of $v$. According to (9), $B_{v}(v, \tau)=0$ if and only if $F\left(x_{1}^{*}\right)=F\left(x_{2}^{*}\right)$. Since $F(x)$ is assumed to be monotone, $F\left(x_{1}^{*}\right)=F\left(x_{2}^{*}\right)$ if and only if $F(x)=k_{1}=$ constant or, equivalently,

$$
H(x) H^{\prime \prime}(x)-k_{1}\left[H^{\prime}(x)\right]^{2}=0 .
$$

$k_{1}$ has to be smaller than one, since otherwise (5) is violated. (13) is a second-order differential equation in $H(x)$. The general solution is obtained as follows. Define $x \geq 0$. According to Szidarovszky and Okuguchi (1997), this is sufficient for the existence of a contest equilibrium. Under the CSF (12), we can explicitly solve for the equilibrium effort levels. It is then straightforward to show that an equilibrium exists, if the contest is not 'too' asymmetric and if $k_{3}$ is not larger than a threshold value. Under the CSF (11), it is not possible to analytically solve for the equilibrium effort levels. But numerical simulations show that there are parameter constellations which ensure existence of an equilibrium. For example, if $k=0.5, v=10, c_{1}=0.5, c_{2}=1.5$ and $\tau=0.5$, then $x_{1}^{*} \approx 1.33, x_{2}^{*} \approx 0.33, \Pi_{x_{1} x_{1}}^{1}\left(x_{1}^{*}, x_{2}^{*}\right) \approx-2.89, \Pi_{x_{2} x_{2}}^{2}\left(x_{2}^{*}, x_{1}^{*}\right) \approx-2.94, \Pi^{1}\left(x_{1}^{*}, x_{2}^{*}\right) \approx 5.08$ and $\Pi^{2}\left(x_{2}^{*}, x_{1}^{*}\right) \approx 2.94$. Details on these computations can be obtained upon request. 
$K:=H^{\prime}$ so that $H^{\prime \prime}=d K / d x=H^{\prime} \cdot(d K / d H)=K \cdot(d K / d H)$. Inserting this into (13) and taking into account $K=H^{\prime}>0$ yields

$$
H \frac{d K}{d H}-k_{1} K=0
$$

which is a first-order differential equation in $K(H)$. If we separate variables, (14) becomes $d K / K=k_{1} \cdot(d H / H)$. Integrating both sides gives $K(H)=k_{2} H^{k_{1}}$ as the general solution to (14). $k_{2}>0$ ensures $K=H^{\prime}>0$. Furthermore, $K=H^{\prime}$ implies $k_{2} H^{k_{1}}=H^{\prime}$ which is a first-order differential equation in $H(x)$. Separating variables, integrating and solving for $H$ yields (12). $k_{3} \geq 0$ ensures $H \geq 0$. Thus, (12) is the general solution to (13) and captures all specifications of $H$ implying $B_{v}(v, \tau)=0$.

An increase in the prize makes the contest more attractive and induces both contestants to increase effort and performance. According to Proposition 2 (a), this is true independently of the functional form of the CSF. In contrast, Proposition 2 (b) shows that the shape of the CSF is crucial for the impact of the prize on competitive balance. If the CSF takes on the special form (10), then a higher prize increases effort levels in such a way that the winning probability of the favorite declines and the winning probability of the underdog rises. Competitive balance is improved. Conversely, if the CSF is represented by (11), an increase in the prize makes it more likely that the favorite wins. Competitive balance deteriorates. Finally, equation (12) fully characterizes the class of CSFs under which winning probabilities and competitive balance are independent of the prize. This independence result is of special interest, since for $k_{1}=(r-1) / r, k_{2}=r$ and $k_{3}=0$ the function (12) encompasses as special case the power CSF $H(x)=x^{r}$ that has frequently been used in the previous literature.

For all CSFs (10) to (12) it is straightforward to show that an increase in the prize raises the favorite's performance by more than the underdog's performance. The difference in performances, $q^{*}=H\left(x_{1}^{*}\right)-H\left(x_{2}^{*}\right)$, increases. But the increase in $q^{*}$ does not necessarily imply a reduction in competitive balance, provided competitive balance is measured by the difference in winning probabilities. The impact of the prize on $b^{*}$ is not unique, but depends on the shape of the CSF. This insight of Proposition 2 is important for the optimal contest design considered in the next sections. 
The comparative static results with respect to the cost parameter $\tau$ are

$$
\begin{aligned}
X_{\tau}^{i}(v, \tau) & =\frac{v\left[H_{i} H_{j}^{\prime \prime}\left(H_{i}+H_{j}\right)-2 H_{i}\left(H_{j}^{\prime}\right)^{2}-H_{i}^{\prime} H_{j}^{\prime}\left(H_{i}-H_{j}\right)\right]}{\Delta\left(H_{i}+H_{j}\right)^{3}}, \quad i \neq j \\
A_{\tau}(v, \tau) & =\frac{v \sum_{i \neq j} H_{i} H_{i}^{\prime}\left[H_{j}^{\prime \prime}\left(H_{i}+H_{j}\right)-2\left(H_{j}^{\prime}\right)^{2}\right]+v H_{1}^{\prime} H_{2}^{\prime}\left(H_{1}-H_{2}\right)\left(H_{2}^{\prime}-H_{1}^{\prime}\right)}{\Delta\left(H_{1}+H_{2}\right)^{3}} \\
& =\frac{v \sum_{i \neq j} H_{i}^{\prime}\left[H_{i} H_{j}^{\prime \prime}-\left(H_{j}^{\prime}\right)^{2}\right]}{\Delta\left(H_{1}+H_{2}\right)^{2}} \\
B_{\tau}(v, \tau) & =\frac{2 v H_{1} H_{2} H_{1}^{\prime} H_{2}^{\prime}}{\Delta\left(H_{1}+H_{2}\right)^{4}}\left[G\left(x_{2}^{*}\right)-G\left(x_{1}^{*}\right)\right]
\end{aligned}
$$

where the subscript $\tau$ denotes the partial derivative with respect to $\tau$ and

$$
G(x):=\frac{H(x) H^{\prime \prime}(x)-\left[H^{\prime}(x)\right]^{2}}{H(x) H^{\prime}(x)} .
$$

Attention is restricted to CSFs implying $G$ to be monotone. (15) to (17) then yield ${ }^{5}$

Proposition 3. (a) An increase in the cost parameter $\tau$ reduces equilibrium effort and performance of the favorite and equilibrium aggregated performance. The effect on equilibrium effort and performance of the underdog is not unique.

(b) An increase in the cost parameter $\tau$ improves competitive balance if $H(x)$ is represented by (10), (11) or (12). It worsens competitive balance if

$$
H(x)=\exp \left\{\int_{0}^{x} \exp \left\{-t^{k}\right\} d t\right\} \quad \text { with } \quad k>1 .
$$

Equilibrium competitive balance is independent of $\tau$ if and only if

$$
H(x)=\exp \left\{\frac{\exp \left\{k_{1}\left(x+k_{3}\right)\right\}+k_{2}}{k_{1}}\right\} \quad \text { with } \quad k_{1}<0, k_{2}, k_{3} \in \mathbb{R} .
$$

\footnotetext{
${ }^{5}$ Under the CSFs (18) and (19) it is again not possible to analytically solve for the equilibrium effort levels. Existence of an equilibrium is therefore demonstrated by way of example. If the CSF is (18) and if $k=2, v=10, c_{1}=0.5, c_{2}=1.5$ and $\tau=0.5$, then $x_{1}^{*} \approx 0.95, x_{2}^{*} \approx 0.45, \Pi_{x_{1} x_{1}}^{1}\left(x_{1}^{*}, x_{2}^{*}\right) \approx-4.29$, $\Pi_{x_{2} x_{2}}^{2}\left(x_{2}^{*}, x_{1}^{*}\right) \approx-2.22, \Pi^{1}\left(x_{1}^{*}, x_{2}^{*}\right) \approx 4.87$ and $\Pi^{2}\left(x_{2}^{*}, x_{1}^{*}\right) \approx 3.35$. If the CSF is (19) and if $k_{1}=-1$, $k_{2}=k_{3}=0, v=10, c_{1}=0.5, c_{2}=1.5$ and $\tau=0.5$, then $x_{1}^{*} \approx 0.87, x_{2}^{*} \approx 0.18, \Pi_{x_{1} x_{1}}^{1}\left(x_{1}^{*}, x_{2}^{*}\right) \approx-3.47$, $\Pi_{x_{2} x_{2}}^{2}\left(x_{2}^{*}, x_{1}^{*}\right) \approx-2.23, \Pi^{1}\left(x_{1}^{*}, x_{2}^{*}\right) \approx 5.16$ and $\Pi^{2}\left(x_{2}^{*}, x_{1}^{*}\right) \approx 3.61$.
} 
Proof: Proposition 1 implies $H_{1}-H_{2}>0$. Using this and (4) in (15) proves $X_{\tau}^{1}(v, \tau)<$ 0 . The sign of $X_{\tau}^{2}(v, \tau)$ is ambiguous. This is shown by way of example. Suppose $H(x)=x^{r}$ and $c_{1}=0.5, c_{2}=3.5, v=10$ and $\tau=0.5$. Then $X_{\tau}^{2}(v, \tau) \approx-0.039$ if $r=0.4$, whereas $X_{\tau}^{2}(v, \tau) \approx 0.020$ if $r=0.8$. To complete the proof of (a), we have to show $A_{\tau}(v, \tau)<0$. Consider first the case $H^{\prime \prime}(x)>0 . x_{1}^{*}>x_{2}^{*}$ then implies $H_{1}>H_{2}$ and $H_{1}^{\prime}>H_{2}^{\prime}$. Taking into account this and (4) in the first line of (16) yields $A_{\tau}(v, \tau)<0$. For $H^{\prime \prime}(x) \leq 0, A_{\tau}(v, \tau)<0$ follows immediately from the second line of (16). In order to prove (b), consider first the CSF (10). Then

$$
G(x)=-\frac{1+\ln (1+x)}{(1+x) \ln (1+x)} \quad \text { and } \quad G^{\prime}(x)=\frac{1+\ln (1+x)+[\ln (1+x)]^{2}}{(1+x)^{2}[\ln (1+x)]^{2}}>0 .
$$

If $H$ satisfies (11), then $G(x)=(k-1) / x$ and $G^{\prime}(x)=(1-k) / x^{2}>0$. For $H$ defined by (12), we obtain $G(x)=-k_{2} /\left(k_{2} x+k_{3}\right)$ and $G^{\prime}(x)=k_{2}^{2} /\left(k_{2} x+k_{3}\right)^{2}>0$. Hence, if $H$ takes on one of the functional forms (10) to (12), then $G\left(x_{1}^{*}\right)>G\left(x_{2}^{*}\right)$ and $B_{\tau}(v, \tau)<0$, i.e. an increase in $\tau$ improves competitive balance. $H$ satisfying (18) implies $G(x)=-k x^{k-1}$ and $G^{\prime}(x)=-k(k-1) x^{k-2}<0$ according to $k>1$. It follows $G\left(x_{2}^{*}\right)>G\left(x_{1}^{*}\right)$ and $B_{\tau}(v, \tau)>0$, i.e. competitive balance deteriorates as $\tau$ increases.

Finally, we have to show under which functional forms of $H$ the parameter $\tau$ does not influence competitive balance. From (17) follows $B_{\tau}(v, \tau)=0$ if and only if $G\left(x_{2}^{*}\right)=$ $G\left(x_{1}^{*}\right)$. Since $G(x)$ is assumed to be monotone, $G\left(x_{2}^{*}\right)=G\left(x_{1}^{*}\right)$ if and only if $G(x)=$ $k_{1}=$ constant. (5) requires $k_{1}<0 . G(x)=k_{1}$ is equivalent to

$$
H(x) H^{\prime \prime}(x)-\left[H^{\prime}(x)\right]^{2}-k_{1} H(x) H^{\prime}(x)=0
$$

which is a second-order differential equation in $H(x)$. The general solution is obtained by defining $K:=H^{\prime}>0$. Taking into account $H^{\prime \prime}=K \cdot(d K / d H),(20)$ becomes

$$
\frac{d K}{d H}-\frac{1}{H} K=k_{1}
$$

which is a first-order differential equation in $K(H)$. Using the integrating factor $\mu:=$ $\exp \left\{-\int d H / H\right\}=1 / H$, the general solution to (21) is

$$
K(H)=\frac{1}{\mu}\left[k_{1} \int \mu d H-k_{2}\right]=k_{1} H \ln H-k_{2} H .
$$

$K=H^{\prime}$ implies $H^{\prime}=k_{1} H \ln H-k_{2} H$. Separating variables, using the transformation $U:=k_{1} \ln H-k_{2}$ with $d U / d H=k_{1} / H$ and integrating yields $U=\exp \left\{k_{1}\left(x+k_{3}\right)\right\}$. 
Resubstituting for $U$ and solving for $H$ gives (19). Hence, (19) is the general solution to (20) and determines all functional forms of $H$ implying $B_{\tau}(v, \tau)=0$.

An increase in the uniform cost parameter makes it more costly for the contestants to perform well in the contest. As a consequence, the favorite reduces effort and performs worse. Although the underdog may increase effort, total performance of both contestants declines. Proposition 3 (a) shows that this is true for every CSF. According to Proposition 3 (b), however, the impact of the cost parameter on competitive balance depends on the functional form of the CSF. If the CSF takes on one of the forms already considered in Proposition 2, then an increase in the cost parameter makes it more likely that the underdog wins. Competitive balance is improved. This result may be seen as intuitively plausible and the only one possible, since an increase in the cost parameter lessens the (relative) cost advantage of the favorite (i.e. $\left(c_{2}+\tau\right) /\left(c_{1}+\tau\right)$ falls). But (19) fully characterizes the class of CSFs under which variations in the cost parameter leave unaltered winning probabilities and competitive balance. Competitive balance may even be worsened by an increase in $\tau$, for example, under the CSF (18).

The insight that a reduction in the cost advantage of the favorite does not necessarily improve competitive balance might be viewed paradoxical. What the decline in the favorite's advantage really induces is a reduction in the difference in performances, $q^{*}=H\left(x_{1}^{*}\right)-H\left(x_{2}^{*}\right)$. This is true for every CSF as can be seen by employing (4), $H_{1}>$ $H_{2}$ and (15). But the decline in the difference in performances does not necessarily come along with an improvement of competitive balance measured by the difference in winning probabilities. Proposition 3 shows that an increase in the cost parameter may leave unchanged or even increase the difference in winning probabilities.

A remark on the relation of Proposition 3 to comparative static results of previous studies is useful. Stein (2002) investigates a contest with $N \geq 2$ players who differ in their abilities. Focusing on the linear CSF, he shows that an increase in contestant $i$ 's relative ability enhances $i$ 's winning probability, while reducing winning probabilities of all other contestants (see his Proposition 5'). Although not explicitly analyzed, an immediate consequence is that competitive balance deteriorates (improves) if $i$ is the strongest (weakest) player. This result is similar to the first statement in Proposition 3 (b). On the one hand, Stein's result is more general than Proposition 3 (b), since he 
allows for more than two contestants. On the other hand, Proposition 3 (b) is more general than the result of Stein, since it is true not only under the linear CSF, but under all CSFs (10) to (12). Proposition 3 (b) also provides CSFs under which the reversed result is obtained. Hence, Stein's result and Proposition 3 (b) complement each other. Furthermore, Baik (1994) analyzes a two-player version of Stein's model. He shows that aggregated effort and performance are maximized in the even contest with both contestants having the same ability. At first glance, this result seems to contradict the finding in Proposition 3 (a) where it has been shown that reducing the advantage of the favorite lessens aggregated effort and performance. But in our model the decline in the favorite's advantage comes from a uniform decrease in the abilities of both contestants, whereas in Baik's model the decline comes from a reduction in the favorite's ability and/or an increase in the underdog's ability. We will further discuss this difference in Section 4 when optimal cost regulation is considered.

\section{Optimal Prize Policy}

Next, the designer's problem of optimally designing the contest is considered. In this section, it is assumed that the designer chooses the prize, but takes the cost parameter as given. Cost regulation is considered in the next sections.

The designer's revenue is denoted by $R(a, b)$. It depends on aggregated performance $a$ and competitive balance $b . R(a, b)$ is supposed to satisfy

$$
\begin{gathered}
R_{a}(a, b)>0, \quad R_{a a}(a, b) \leq 0, \\
R_{b}(a, b) \gtreqless 0 \Leftrightarrow b \lesseqgtr 0, \quad R_{b b}(a, b)<0 .
\end{gathered}
$$

Equation (22) states that an increase in the performance of the contestants enhances the designer's revenue at non-increasing rates. According to (23), the revenue function is inverted U-shaped with respect to the difference in winning probabilities with a maximum at the point where the difference vanishes. Improving competitive balance therefore increases the designer's revenue and, for given performance, revenue is maximized in the even contest with both contestants having the same chance of winning.

The designer maximizes profit with respect to the prize $v$. In doing so, she takes into 
account the impact of the prize on aggregated performance and competitive balance in the contest equilibrium. The designer's maximization problem reads

$$
\max _{v} \Pi(v)=R[A(v), B(v)]-v
$$

where $\tau$ is suppressed in $A(v)$ and $B(v)$, since in this section it is taken as given. The first-order condition for the designer's profit maximum is

$$
\Pi_{v}(v)=0 \quad \Leftrightarrow \quad R_{a}[A(v), B(v)] A_{v}(v)+R_{b}[A(v), B(v)] B_{v}(v)=1 .
$$

A marginal increase in the prize influences the designer's profit through three channels. First, it increases the designer's cost. This marginal cost is represented by the RHS of (25). Second, it makes the contest more attractive and increases effort and performance of the contestants such that the designer's revenue is enhanced. This marginal revenue is captured by $R_{a}(\cdot) A_{v}(v)>0$ in (25). Third, it may also alter the designer's revenue through a change in competitive balance. This effect is represented by $R_{b}(\cdot) B_{v}(v)$ in (25). $R_{b}(\cdot)$ is negative, because the difference in winning probabilities is positive in the contest equilibrium. Hence, if the marginal increase in the prize improves competitive balance $\left(B_{v}<0\right)$, then revenue is enhanced and $R_{b}(\cdot) B_{v}(v)>0$ represents marginal revenue. In case the marginal increase in the prize reduces competitive balance $\left(B_{v}>\right.$ 0 ), revenue declines and $R_{b}(\cdot) B_{v}(v)<0$ represents marginal cost. If the prize increase does not affect competitive balance $\left(B_{v}=0\right)$, then the marginal effect $R_{b}(\cdot) B_{v}(v)$ vanishes. In sum, condition (25) states that all marginal effects of the prize have to be equalized in the profit maximum of the designer.

Of special interest is how the inclusion of competitive balance in the objective of the designer influences the optimal prize. We therefore compare the case in which the revenue function satisfies (23) with the case in which the revenue function does not depend on competitive balance, i.e. $R_{b} \equiv 0$. The result is contained in

Proposition 4. Suppose $R_{b}$ satisfies (23). If $H(x)$ takes on the functional form (10) $((11))$, then the optimal price $v^{*}$ is larger (smaller) than in case of $R_{b} \equiv 0$. The optimal price $v^{*}$ is the same as in case of $R_{b} \equiv 0$ if and only if $H(x)$ satisfies (12).

Proof: The optimal prize $v^{*}$ is determined by (25). This equation can be written as

$$
R_{a}\left[A\left(v^{*}\right), B\left(v^{*}\right)\right] A_{v}\left(v^{*}\right)+\delta R_{b}\left[A\left(v^{*}\right), B\left(v^{*}\right)\right] B_{v}\left(v^{*}\right)-1=0 .
$$


For $\delta=1$ we are in the case where $R_{b}$ satisfies (23). $\delta=0$ simulates the case $R_{b} \equiv 0$. Implicitly differentiating (26) yields

$$
\frac{d v^{*}}{d \delta}=-\frac{R_{b}(\cdot) B_{v}\left(v^{*}\right)}{\Pi_{v v}}
$$

where $R_{b}(\cdot)<0$ owing to $b^{*}>0$. $\Pi_{v v}$ has to be negative according to the secondorder condition of the designer's profit maximization. If $H(x)$ is represented by (10), Proposition 2 shows that $B_{v}<0$ and (27) implies $d v^{*} / d \delta>0$, i.e. the optimal prize in the case where $R_{b}$ satisfies (23) is larger than in the case $R_{b} \equiv 0$. The reversed argument applies if $H(x)$ is represented by (11). Finally, $d v^{*} / d \delta=0$ is equivalent to $B_{v}=0$. According to Proposition 2, $B_{v}=0$ if and only if $H(x)$ satisfies (12).

The intuition of Proposition 4 is as follows. If the CSF is represented by (10), then we know from Proposition 2 that a marginal increase in the prize improves both aggregated performance and competitive balance. The revenue of the designer is therefore increased not only because the contestants perform better, but also because the contest becomes closer. The designer accounts for this additional positive effect of a prize increase and chooses a larger prize than in the case where her objective does not depend on competitive balance. This argument is reversed if the CSF takes on the functional form (11). For the class of CSFs described by (12), a marginal increase in the prize leaves unaltered the winning probabilities and competitive balance. Hence, the optimal prize setting behavior of the designer is as if her revenue does not depend on competitive balance. This independence result is of special interest, since (12) comprises as special case the often used power CSF $H(x)=x^{r}$.

The findings in Proposition 4 contrast the result of Singh and Wittman (1998). They show that the inclusion of competitive balance in the objective of the contest designer reduces the optimal prize. The reason for this difference in results lies in the measure of competitive balance. If we follow Singh and Wittman (1998) and measure the closeness of the contest by the difference in performances, then the optimal prize in our model is also unambiguously reduced by the inclusion of competitive balance. Remember that an increase in the prize raises the difference in performance independently of the functional form of the CSF (see p. 8). But the impact of the prize on the difference in winning probabilities depends on the shape of the CSF. Using this difference as measure of competitive balance therefore generates ambiguous results. 


\section{Optimal Cost Regulation}

Suppose now the designer takes as given the prize and instead regulates the contestants' costs. Since she cares about competitive balance, the designer might want to make the contest closer by treating the contestants differently, i.e. by increasing the cost of the favorite and reducing that of the underdog. But in our model the designer controls the uniform cost parameter $\tau$ only and treats contestants equally. This assumption reflects the idea that the designer is not able to discriminate between contestants. A reason may be, for example, that the audience cannot observe the contestants' costs and therefore regards a unequal treatment unfair. Moreover, our contest with asymmetric costs is equivalent to a contest with asymmetric abilities where an increase in $\tau$ harms the contestants' abilities. Hence, $\tau$ can be interpreted as a parameter representing the rules of the contest. These rules are usually the same for all contestants.

When the prize is taken as given, the designer maximizes profit by maximizing revenue. The associated optimization problem reads

$$
\max _{\tau} \Pi(\tau)=R[A(\tau), B(\tau)] \quad \text { s.t. } \quad \tau \geq 0
$$

For two reasons the cost parameter is supposed to be non-negative. First, allowing negative values of $\tau$ causes existence problems. The designer can always increase profit by reducing the contestants' costs, because performance is then improved. Second, increasing the contestants' costs is often easier for the designer than reducing the costs. For example, in motor racing it is straightforward to restrict the technically properties of the cars such that their performance is reduced, but it is far more difficult to provide the teams with performance improving appliances. The Kuhn-Tucker firstorder conditions for the solution of (28) are

$$
\Pi_{\tau}(\tau)=R_{a}[A(\tau), B(\tau)] A_{\tau}(\tau)+R_{b}[A(\tau), B(\tau)] B_{\tau}(\tau) \leq 0, \quad \tau \Pi_{\tau}(\tau)=0, \quad \tau \geq 0
$$

A marginal increase in the parameter $\tau$ reduces the designer's profit by a decline in aggregated performance of the contestants. This marginal cost of $\tau$ is represented by $R_{a}(\cdot) A_{\tau}(\tau)<0$ in (29). Furthermore, the marginal increase in $\tau$ may influence the designer's profit through a change in competitive balance. This effect is represented by $R_{b}(\cdot) B_{\tau}(\tau)$ in $(29)$. Remember that $R_{b}(\cdot)$ is always negative due to $b^{*}>0$. Hence, 
if competitive balance is improved as $\tau$ increases $\left(B_{\tau}<0\right)$, then the designer's revenue increases and $R_{b}(\cdot) B_{\tau}(\tau)>0$ represents marginal revenue of $\tau$. The reversed is true if competitive balance is worsened $\left(B_{\tau}>0\right)$ such that $R_{b}(\cdot) B_{\tau}(\tau)<0$ indicates marginal cost of $\tau$. If changes in $\tau$ do not influence competitive balance, then $R_{b}(\cdot) B_{\tau}(\tau)=0$ and the designer's revenue is altered through changes in aggregated performances only, but not through changes in competitive balance.

It is again of special interest how the inclusion of competitive balance in the designer's objective influences the optimal contest design. This question is answered in the next proposition, where $\tau^{*}$ denotes the optimal value of the cost parameter.

Proposition 5. $R_{b} \equiv 0$ implies $\tau^{*}=0$. The same holds if $R_{b}$ satisfies (23) and $H(x)$ is represented by $(18)$ or $(19)$. If $R_{b}$ satisfies $(23)$ and $H(x)$ is represented by $(10),(11)$ or (12), then $\tau^{*}$ may be positive.

Proof: Inserting $R_{b} \equiv 0$ into (29) implies $\Pi_{\tau}<0$, since $R_{a}(\cdot)>0$ and $A_{\tau}<0$ according to (22) and Proposition 3 (a). The slackness condition in (29) then yields $\tau^{*}=0$ which proves the first part of Proposition 5. If $H(x)$ is defined by (18) or (19), we know from Proposition 3 (b) that $B_{\tau} \geq 0$. Owing to $R_{b}(\cdot)<0$, (29) again implies $\Pi_{\tau}<0$ and $\tau^{*}=0$. It remains to show that $\tau^{*}$ may be positive if $H(x)$ is represented by (10), (11) or (12). Under these functional forms of $H$, Proposition 3 (b) shows that $B_{\tau}<0$. Consequently, $\Pi_{\tau}$ in (29) is not necessarily smaller than zero and $\tau^{*}$ may become positive. This can be proven by way of example. Suppose $H(x)=x$. Equilibrium aggregate performance and equilibrium competitive balance can then be computed from (3) as

$$
A(\tau)=\frac{v}{c_{1}+c_{2}+2 \tau}, \quad B(\tau)=\frac{c_{2}-c_{1}}{c_{1}+c_{2}+2 \tau}
$$

If $R(a, b)=\alpha a-\beta b^{2}$ with $\alpha, \beta>0$, then the cost parameter $\tilde{\tau}$ satisfying $\Pi_{\tau}(\tilde{\tau})=0$ is

$$
\tilde{\tau}=\frac{\beta\left(c_{2}-c_{1}\right)^{2}}{\alpha v}-\frac{c_{1}+c_{2}}{2}
$$

If the model parameters satisfy $2 \beta\left(c_{2}-c_{1}\right)^{2}>\alpha v\left(c_{1}+c_{2}\right)$, then $\tau^{*}=\tilde{\tau}>0$.

According to Proposition 5, there is no scope for cost regulation if the designer's revenue depends on performance of the contestants only. Increasing the cost parameter to a positive value would harm performance and unambiguously reduce the designer's profit. 
The picture is less clear in case the designer additionally cares about competitive balance. If the CSF is (18) or (19), then we know from Proposition 3 (b) that an increase in the cost parameter does not improve competitive balance. Hence, the designer still cannot profit from cost regulation. In contrast, if the CSF takes on one of the functional forms (10) to (12), then Proposition 3 (b) states that the contest becomes closer as the cost parameter increases. Consequently, the designer has to balance the negative effect of reducing performance of the contestants and the positive effect of improving competitive balance. If the latter effect is strong enough, it is rational for the designer to shift the cost parameter to a positive value. This case is of special interest, since it is true also for the often used power CSF $H(x)=x^{r}$.

Proposition 5 provides the rationale for cost regulation by way of example 'only'. It is therefore desirable to gather some information about the condition under which such a result prevails. We do this by conducting a comparative static analysis in case of the linear CSF and the linear-quadratic revenue function already employed in the proof of Proposition 5. Differentiating (31) immediately yields

Proposition 6. Suppose $H(x)=x$ and $R(a, b)=\alpha a-\beta b^{2}$ with $\alpha, \beta>0$. Then $\tau^{*}>0$ is the more likely, the larger is $\beta$, the smaller is $\alpha$ and/or the larger is $c_{2}-c_{1}$.

To understand the intuition of this result, remember that under the linear CSF an increase in the cost parameter reduces aggregated performance $\left(A_{\tau}<0\right)$ and improves competitive balance $\left(B_{\tau}<0\right)$. If $\beta$ increases and/or $\alpha$ decreases, then the designer cares more about competitive balance and less about aggregated performance. Hence, she places more weight on the positive effect of cost regulation on competitive balance and less weight on the negative effect of cost regulation on aggregated performance. As a consequence, it becomes more likely that the designer chooses a positive value for the cost parameter. If the cost differential $c_{2}-c_{1}$ is increased, competitive balance in the contest equilibrium deteriorates. The designer compensates this negative effect by increasing the cost parameter, because this partly offsets the increase in the cost advantage of the favorite and renders the contest closer again. Hence, the more different contestants are, the more likely is that the designer employs cost regulation. 


\section{Optimal Mix of Prize Policy and Cost Regulation}

Let us finally turn to the most realistic case where the designer sets both the prize and the cost parameter. Her profit maximization problem then reads

$$
\max _{v, \tau} \Pi(v, \tau)=R[A(v, \tau), B(v, \tau)]-v \quad \text { s.t. } \quad \tau \geq 0 .
$$

The Kuhn-Tucker first-order conditions can be written as

$$
\begin{gathered}
\Pi_{v}(v, \tau)=R_{a}(\cdot) A_{v}(v, \tau)+R_{b}(\cdot) B_{v}(v, \tau)-1=0 \\
\Pi_{\tau}(v, \tau)=R_{a}(\cdot) A_{\tau}(v, \tau)+R_{b}(\cdot) B_{\tau}(v, \tau) \leq 0, \quad \tau \Pi_{\tau}(v, \tau)=0, \quad \tau \geq 0 .
\end{gathered}
$$

These conditions reflect the different channels through which changes in the prize and the cost parameter influence the designer's profit. With respect to the optimal cost parameter, we obtain the same result as in Proposition 5. If the designer's revenue does not depend on competitive balance $\left(R_{b} \equiv 0\right)$ or if an increase in the cost parameter does not improve competitive balance $\left(B_{\tau} \geq 0\right)$, then (34) yields the corner solution $\tau^{*}=0$, i.e. cost regulation is not optimal. In such cases, the optimal prize $v^{*}$ is determined by equation (33) which is the same as in Section 3. In contrast, if the revenue function depends on competitive balance ( $R_{b}$ satisfies (23)) and competitive balance is improved as the contestants' costs are regulated $\left(B_{\tau}<0\right), \tau^{*}$ may be positive. The optimal prize and the optimal cost parameter are then simultaneously determined by equations (33) and (34), where in (34) the strict equality sign prevails.

For such a case, it will finally be investigated how changes in the model parameter affect the designer's optimal mix of prize policy and cost regulation.

Proposition 7. Suppose $H(x)=x, R(a, b)=\alpha \sqrt{a}-\beta b^{2}$ with $\alpha, \beta>0$ and $\tau^{*}>0$. Then, $\tau^{*}$ is increasing in $\beta$ and in $c_{2}-c_{1}$, but decreasing in $\alpha . v^{*}$ is decreasing in $\beta$ and in $c_{2}-c_{1}$, but increasing in $\alpha$.

Proof: For $H(x)=x$, equilibrium aggregated performance and equilibrium competitive balance are captured by (30). $\tau^{*}>0$ implies that the first condition in (34) holds as equality. $v^{*}$ and $\tau^{*}$ can then explicitly be determined by computing the derivatives of (30) and using $R(a, b)=\alpha \sqrt{a}-\beta b^{2}$ in (33) and (34). The result is

$$
v^{*}=\frac{\alpha^{4}}{32 \beta\left(c_{2}-c_{1}\right)^{2}}, \quad \tau^{*}=\frac{4 \beta\left(c_{2}-c_{1}\right)^{2}}{\alpha^{2}}-\frac{c_{1}+c_{2}}{2} \text {. }
$$


Differentiating with respect to $\beta, \alpha$ and $c_{2}-c_{1}$ immediately proves Proposition 7 .

To understand this result, remember that under the linear CSF an increase in the prize enhances aggregated performance $\left(A_{v}>0\right)$ while leaving unchanged competitive balance $\left(B_{v}=0\right)$, and an increase in the cost parameter reduces aggregated performance $\left(A_{\tau}<0\right)$ while improving competitive balance $\left(B_{\tau}<0\right)$. If $\beta$ increases and/or $\alpha$ decreases, then the designer is concerned more with competitive balance and less with the performance of the contestants. Hence, she reduces the cost advantage of the favorite and renders the contest closer through a higher value of the cost parameter. Since performance is less important, she also reduces her cost by decreasing the prize. If $c_{2}-c_{1}$ increases, competitive balance in the contest equilibrium declines. The designer should then be more concerned with competitive balance and, thus, she tightens cost regulation and reduces the prize.

These results are obtained by focusing on the linear CSF. But the optimal cost parameter may be positive under all CSFs (10) to (12). Unfortunately, under these functional forms the optimal contest design is analytically not tractable. But under the CSF (12), the same results as in Proposition 7 are expected, since the effects of the prize and the cost parameter on aggregated performance and competitive balance are the same as for the linear CSF. It should also be possible to extent Proposition 7 to the CSF (11). The only difference to the linear CSF is that an increase in the prize worsens competitive balance $\left(B_{v}>0\right)$. Hence, as a reaction on an increase in $\beta$ and $c_{2}-c_{1}$ or a decrease in $\alpha$, the designer reduces the prize not only because this lowers her cost, but also because the contest becomes closer. Under the CSF (10), a prize increase improves competitive balance $\left(B_{v}<0\right)$ and, consequently, it is no longer clear how the designer reacts on changes in the model parameters.

\section{Conclusion}

This paper investigates optimal contest design in case the designer's objective function depends on competitive balance. The optimal prize policy and optimal cost regulation have been derived and compared with the case the designer's objective is not influenced by competitive balance. In contrast to previous models, the effect of competitive balance on the optimal prize has been shown to depend on the shape of the 
CSF. Perhaps most interestingly, under the frequently used power CSF the inclusion of competitive balance in the designer's objective does not influence the optimal prize. With respect to optimal cost regulation, it has been shown that it may become optimal for the designer to increase the contestant's effort costs (to decrease their abilities), if competitive balance is an argument of the designer's objective function.

The analysis proceeded on some assumptions which can serve as starting point for further research. For example, the analysis in this paper considers simultaneous move contests only. Both contestants simultaneously choose their effort levels. In many contest, however, effort levels are chosen sequentially. Furthermore, we focused on a two-player contest. But many contests have more than two contestants. It may therefore be interesting to find out whether and, if so, how our results change in a contest with sequential decision of the contestants and/or in a multi-player contest.

\section{References}

Amegashie, J.A. (1999), 'The Design of Rent-Seeking Competitions: Committees, Preliminary and Final Contests', Public Choice 99, 63-76.

Amegashie, J.A. (2000), 'Some Results on Rent-Seeking Contests with Shortlisting', Public Choice 105, 245-253.

Baik, K.H. (1994), 'Effort Levels in Contests with Two Asymmetric Players', Southern Economic Journal 61, 367-378.

Dasgupta, A., and K.O. Nti (1998), 'Designing an Optimal Contest', European Journal of Political Economy 14, 587-603.

Fort, R. and J. Quirk (1995), 'Cross Subsidization, Incentives and Outcomes in Professional Team Sports Leagues', Journal of Economic Literature XXXIII, 1265-1299.

Glazer, A., and R. Hassin (1988), 'Optimal Contests', Economic Inquiry 26, 133-143.

Gradstein, M. (1998), 'Optimal Contest Design: Volume and Timing of Rent Seeking Contests', European Journal of Political Economy 14, 575-585. 
Gradstein, M., and K.A. Konrad (1999), 'Orchestrating Rent-Seeking Contests', Economic Journal 109, 536-545.

Kohli, I., and N. Singh (1999), 'Rent Seeking and Rent Setting with Asymmetric Effectiveness of Lobbying', Public Choice 99, 275-298.

Kohli, I., and N. Singh (2001), 'A Model of Inequality and Interest Group Politics', Economics and Politics 13, 185-200.

Nti, K.O. (1999a), 'Stability in the Patent Race Contest of Lee and Wilde', Economic Theory 14, 237-245.

Nti, K.O. (1999b), 'Rent-Seeking with Asymmetric Valuations', Public Choice 98, 415-430.

Singh, N., and D. Wittman (1988), 'Economic Contests with Incomplete Information', Management Science 34, 528-540.

Singh, N., and D. Wittman (1998), 'Contest Design and the Objective of the Contest Designer: Sales Promotion, Sporting Events, and Patent Races', in: Baye, M. (ed.), Advances in Applied Microeconomics Vol. 7, JAI Press, 139-167.

Singh, N., and D. Wittman (2001), 'Contests where there is Variation in the Marginal Productivity of Effort', Economic Theory 18, 711-744.

Skarperdas, S. (1996), 'Contest Success Functions', Economic Theory 7, 283-290.

Stein, W.E. (2002), 'Asymmetric Rent-Seeking with More Than Two Contestants', Public Choice 113, 325-336.

Szidarovszky, F., and K. Okuguchi (1997), 'On the Existence and Uniqueness of Pure Nash Equilibrium in Rent-Seeking Games', Games and Economic Behavior 18, 135-140.

Szymanski, S. (2003), 'The Economic Design of Sporting Contests: A Review', forthcoming Journal of Economic Literature. 
Szymanski, S. and T. Valletti (2002), 'First and Second Prizes in Imperfectly Discriminating Contests', Mimeo.

Tullock, G. (1980), 'Efficient Rent-Seeking', in: Buchanan, J.M., Tollison, R.D., and G. Tullock (eds.), Toward a Theory of the Rent-Seeking Society, College Station: Texas A\& M University Press. 